\title{
Cognitive Impairment and Psychological Distress at Discharge from Intensive Care Unit
}

\author{
Chi Ryang Chung1', Hye Jin Yoo², Jinkyeong Park¹, and Seunghyong Ryu ${ }^{2} \bowtie$ \\ ${ }^{1}$ Department of Critical Care Medicine, Samsung Medical Center, Sungkyunkwan University School of Medicine, Seoul, Republic of Korea \\ ${ }^{2}$ Department of Psychiatry, Samsung Medical Center, Sungkyunkwan University School of Medicine, Seoul, Republic of Korea
}

This study aimed to investigate cognitive impairment and psychological distress of critically ill patients at discharge from intensive care unit (ICU). This study included 30 critically ill patients who had neither pre-existing dementia nor ongoing delirium. At ICU discharge, they performed a screening test for cognitive impairment (Mini-Cog test) and completed questionnaires for depression (Patient Health Questionnaire-2, PHQ-2) and for 4 stressful experiences during ICU stay including nightmares, severe anxiety or panic, severe pain, and trouble to breathe or feeling of suffocation (Post-Traumatic Stress Syndrome 14-Question Inventory, PTSS-14 Part A). Thirteen patients (43.3\%) screened positive for cognitive impairment and 18 patients $(60.0 \%)$ exhibited depressive symptoms. Twenty three patients (76.7\%) recollected one or more stressful in-ICU experiences. Female patients (88.9\%) was more likely to feel depressed at ICU discharge, compared to male patients $(47.6 \%)\left(\chi^{2}=4.47, \mathrm{p}=0.03\right)$. To the best of our knowledge, this is the first report on cognitive and psychological outcomes of ICU survivors in Korea. In this study, we observed that a considerable number of critically ill patients had experienced cognitive impairment or psychological distress at ICU discharge.

Psychiatry Investig 2017;14(3):376-379

Key Words Critical care, Cognitive impairment, Psychological distress, Post-intensive care syndrome.

\section{INTRODUCTION}

Critically ill patients experience extreme levels of physical and psychological stresses in the intensive care unit (ICU). These experiences may result in long-term cognitive or psychological morbidity in patients surviving critical illness, known as post-intensive care syndrome (PICS). ${ }^{1}$ Cognitive decline occurs in up to $78 \%$ of survivors one year after ICU discharge. ${ }^{2}$ Systematic reviews of long-term psychological morbidity in general ICU survivors found that the point prevalence of clinically significant depressive symptoms and PTSD symptoms was $28 \%$ and $22 \%$, respectively. ${ }^{3,4}$ The new or worsening impairments in cognition and mental health after critical illness may lead to reduced quality of life. ${ }^{5}$ Given the large number of acutely ill patients being treated in the ICU every year, long-term cognitive or psychological morbidity after

Received: January 6, 2016 Revised: March 15, 2016

Accepted: May 17, 2016 Available online: February 4, 2017

$\triangle$ Correspondence: Seunghyong Ryu, MD

Department of Psychiatry, Samsung Medical Center, Sungkyunkwan University School of Medicine, 81 Irwon-ro, Gangnam-gu, Seoul 06351, Republic of Korea Tel: +82-2-3410-3589, Fax: +82-2-3410-0050

E-mail: seunghyongryu@gmail.com

(a) This is an Open Access article distributed under the terms of the Creative Commons Attribution Non-Commercial License (http://creativecommons.org/licenses/bync/4.0) which permits unrestricted non-commercial use, distribution, and reproduction in any medium, provided the original work is properly cited. critical illness may be a growing public health problem.

It is important to detect these psychiatric problems early following critical illness and to determine the risk of future cognitive and psychological morbidity. ${ }^{67}$ However, until now, most ICU survivors in Korea are discharged from the hospital without assessment of cognitive impairment and psychological distress following critical illness, and some of them might continue to suffer from long-term cognitive or psychological morbidity. Researchers in the US and UK have investigated post-ICU physical, cognitive, and mental health problems more than 10 years ago. ${ }^{2-4}$ Some studies had been performed to detect the patients at high risk for cognitive impairment after critical care and recent researches assessing the effect of early rehabilitation on long-term cognitive outcomes are underway., ${ }^{8,9}$ The 2009 National Institute for Health and Clinical Excellence (NICE) guideline stated that patients should be assessed in the ICU for acute distress and risk of future psychological morbidity. ${ }^{10}$ However, in Korea, little is known about how many patients have experienced cognitive or psychological problems after ICU discharge. In this retrospective study, we assessed the acute impact of critical care on cognitive function and mental health and investigated its association with clinical ICU data in Korean ICU survivors. To the best of our knowledge, this is the first report on cognitive 
and psychological outcomes of ICU survivors in Korea.

\section{METHODS}

In this study, we reviewed the medical records of 43 patients who were referred to the psychiatric consultation service from June 2014 to May 2015 for the evaluation of cognitive impairment or mood disturbances at discharge from the medical intensive care unit (MICU), coronary care unit (CCU), or surgical intensive care unit (SICU) of Samsung Medical Center, a 1960-bed, university-affiliated, tertiary referral hospital in Seoul, Korea. Following the routine protocol for psychiatric evaluation of critically ill patients, the patients were assessed for ongoing delirium with the Confusion Assessment Method for the ICU (CAM-ICU) ${ }^{11}$ and for pre-existing dementia with the Short Form of the Samsung Dementia Questionnaire (S-SDQ) ${ }^{12}$ at ICU discharge. They also underwent a screening test for cognitive impairment (Mini-Cog test $)^{13,14}$ and completed questionnaires for depressive symptoms (Patient Health Questionnaire-2, PHQ-2) ${ }^{15}$ and for 4 stressful experiences during ICU stay including nightmares, severe anxiety or panic, severe pain, and difficulty in breathing or feeling of suffocation (Post-Traumatic Stress Syndrome 14-Question Inventory, PTSS-14 Part A). ${ }^{16}$ Among them, 30 patients who had neither ongoing delirium (CAM negative) nor pre-existing dementia (S-SDQ score $<8$ ) were included in the analysis. The point prevalence of cognitive impairment, depressive symptoms, and distressing memories of the ICU stay was analyzed with descriptive statistics. In addition, we investigated its association with demographic and clinical data which were obtained from chart review, using the chi-square test or Mann-Whitney U test. The Institutional Review Board of Samsung Medical Center approved the review of information obtained from the patients' records. The requirement of informed consent was waived because of the retrospective nature of the study.

\section{RESULTS}

Characteristics of the patients during the ICU stay are presented in Table 1. On the Mini-Cog test, 13 patients (43.3\%) screened positive for cognitive impairment [recall of 0 item $(n=8)$ or recall of 1-2 items with an abnormal clock face $(n=5)]$. Although not statistically significant, patients with cognitive impairment at ICU discharge were more likely to have a higher Sequential Organ Failure Assessment (SOFA) score at ICU admission $(U=70.00, p=0.09)$. Eighteen patients (60.0\%) expressed depressive symptoms with a PHQ-2 score $\geq 3$ (median PHQ-2 score 3, IQR 1-5). Female patients (88.9\%) were more likely to feel depressed at ICU discharge, com-
Table 1. Demographic and clinical characteristics

\begin{tabular}{lc}
\hline \multicolumn{1}{c}{ Variables } & $\begin{array}{c}\text { No. of patients (\%) } \\
\text { or median (IQR) }\end{array}$ \\
\hline Age, y & $61(50-72)$ \\
Sex, female & $9(30.0)$ \\
$<$ High school graduate & $5(16.7)$ \\
Type of ICU & \\
Medical ICU & $22(73.3)$ \\
Coronary Care Unit & $2(6.7)$ \\
Surgical ICU & $6(20.0)$ \\
Major reasons for ICU admission & \\
Cardiovascular & $2(6.7)$ \\
Respiratory & $17(56.7)$ \\
Endocrine & $1(3.3)$ \\
Severe sepsis or septic shock & $3(10.0)$ \\
Postoperative & $7(23.3)$ \\
GCS score at ICU admission & $14(10-15)$ \\
SOFA score at ICU admission & $7(3-10)$ \\
Duration of mechanical ventilation, d & $5.5(0-14)$ \\
Duration of delirium, d & $4(0-9)$ \\
ICU length of stay, d & $11(7-16)$ \\
\hline
\end{tabular}

IQR: interquartile range, ICU: intensive care unit, GCS: glasgow coma scale, SOFA: sequential organ failure assessment

pared to male patients $(47.6 \%)\left(\chi^{2}=4.47, p=0.03\right)$. With regard to memories of the ICU stay, 23 patients (76.7\%) recollected one or more stressful in-ICU experiences including nightmares $(n=9,30.0 \%)$, severe anxiety or panic $(n=18,60.0 \%)$, severe pain $(n=9,30.0 \%)$, and difficulty in breathing or feeling of suffocation $(\mathrm{n}=13,43.3 \%)$. We observed trends suggesting that longer ICU length of stay was associated with postICU memory of severe anxiety or panic during the ICU stay $(\mathrm{U}=66.00, \mathrm{p}=0.08)$ and longer duration of mechanical ventilation was associated with post-ICU memory of difficulty in breathing or feeling of suffocation during the ICU stay ( $\mathrm{U}=$ $69.50, \mathrm{p}=0.09$ ).

\section{DISCUSSION}

Acute impact of critical illness on cognitive function has not been deeply studied. To the best of our knowledge, two studies have assessed cognitive function of non-delirious patients at ICU discharge. Jones et al. ${ }^{17}$ described that 26 of 30 patients $(86.7 \%)$ showed significant problems with strategic thinking and problem solving 1 week after ICU discharge. Torgersen et al. ${ }^{18}$ reported that 18 of 28 patients (64.3\%) had a cognitive impairment at ICU discharge. These studies used the Cambridge Neuropsychological Test Automated Battery (CANTAB), ${ }^{19}$ a computerized neuropsychological test battery, 
and assessed cognitive function of patients across multiple cognitive domains. On the other hand, in the current study, only $43.3 \%$ of non-demented and non-delirious patients who were just discharged from the ICU screened positive for cognitive impairment. Because ICU survivors often find it difficult to complete neuropsychological tests immediately after discharge from the ICU due to their poor physical condition, we used the Mini-Cog test, a very simple screening tool consisting of 3-item recall and clock-drawing, to minimize the burden on patients. Considering that the sensitivity and specificity of the Mini-Cog test for cognitive impairment are known to be comparable to those achieved using conventional neuropsychological test batteries, ${ }^{13,14}$ the difference in the prevalence can be explained by differences in test performance of patients. In addition, we did not find any association between cognitive impairment at ICU discharge and its risk factors, including age ( $\mathrm{U}=86.00, \mathrm{p}=0.32)$, pre-existing cognitive impairment (S-SDQ score) $(\mathrm{U}=103.00, \mathrm{p}=0.77)$, and duration of delirium in the ICU ( $\mathrm{U}=84.00, \mathrm{p}=0.28)$, but we only observed a trend for association with the initial severity of illness (SOFA score at ICU admission) $(\mathrm{U}=70.00, \mathrm{p}=0.09)$. Several in-ICU illness factors including hypoxia and hypotension, sepsis, and dysglycemia have been identified as risk factors for cognitive impairment following critical illness. ${ }^{2}$

Early post-ICU depressive symptoms and post-ICU memories of in-ICU experiences have been shown to be the strongest risk factors for long-term depression and PTSD, respectively, ${ }^{3,4}$ and therefore, ICU survivors need to be screened for early psychological distress following critical illness no later than the day of hospital discharge to minimize future psychological morbidities. Because general depression or PTSD screening tools are not suitable for assessing severely ill patients, we simply examined whether the patients who were just discharged from the ICU had been bothered by depressed mood or loss of interest and memories of in-ICU stressful experiences, using very short questionnaires such as PHQ-2 and PTSS-14 Part A. In this study, more than half of the patients exhibited depressive symptoms at ICU discharge and this rate seems to be higher than the prevalence rate of depression at hospital discharge in previous studies (i.e., 14$36 \%){ }^{3}$ This discrepancy might be due to differences in measurement time or screening tools. We also observed that about three-fourths of the patients had one or more stressful memories of in-ICU experiences at ICU discharge, and this rate is comparable to that reported by Samuelson et al. ${ }^{20}$ In addition, we found a significant association between postICU depressive symptoms and female sex, and trends of association between post-ICU memories and ICU length of stay and duration of mechanical ventilation. These demographic and in-ICU illness and treatment factors had been identified as significant predictors of post-ICU depression or PTSD in some previous studies. ${ }^{3,4}$

There are some limitations to this study. First, as the sample size was small, we might not have been able to detect a significant effect of probable risk factors on cognitive and psychological outcomes. Second, since it was a retrospective study, some selection bias might have affected the results. Third, some important variables (severity of delirium and exposure to sedative and analgesic medications) could not be included in the current analysis. Therefore, our results should be interpreted as preliminary findings. However, it is meaningful that we could assess cognitive function and psychological status of critically ill patients at ICU discharge, using simple screening tools to which even severely ill patients can easily respond. In the current study, we observed that a considerable number of patients had experienced cognitive impairment or psychological distress at ICU discharge. Further prospective studies are warranted to investigate the shortand long-term cognitive and psychological morbidities following critical illness and their risk factors in Korean ICU survivors.

\section{REFERENCES}

1. Needham DM, Davidson J, Cohen H, Hopkins RO, Weinert C, Wunsch $\mathrm{H}$, et al. Improving long-term outcomes after discharge from intensive care unit: report from a stakeholders' conference. Crit Care Med 2012;40:502-509.

2. Wilcox ME, Brummel NE, Archer K, Ely EW, Jackson JC, Hopkins RO. Cognitive dysfunction in ICU patients: risk factors, predictors, and rehabilitation interventions. Crit Care Med 2013;41(9 Suppl 1): S81-S98.

3. Davydow DS, Gifford JM, Desai SV, Bienvenu OJ, Needham DM. Depression in general intensive care unit survivors: a systematic review. Intensive Care Med 2009;35:796-809.

4. Davydow DS, Gifford JM, Desai SV, Needham DM, Bienvenu OJ. Posttraumatic stress disorder in general intensive care unit survivors: a systematic review. Gen Hosp Psychiatry 2008;30:421-434.

5. Davidson JE, Harvey MA, Bemis-Dougherty A, Smith JM, Hopkins RO. Implementation of the Pain, Agitation, and Delirium Clinical Practice Guidelines and promoting patient mobility to prevent post-intensive care syndrome. Crit Care Med 2013;41(9 Suppl 1):S136-S145.

6. Peris A, Bonizzoli M, Iozzelli D, Migliaccio ML, Zagli G, Bacchereti A, et al. Early intra-intensive care unit psychological intervention promotes recovery from post traumatic stress disorders, anxiety and depression symptoms in critically ill patients. Crit Care 2011;15:R41.

7. Wade DM, Hankins M, Smyth DA, Rhone EE, Mythen MG, Howell DC, et al. Detecting acute distress and risk of future psychological morbidity in critically ill patients: validation of the intensive care psychological assessment tool. Crit Care 2014;18:519.

8. Brummel NE, Girard TD, Ely EW, Pandharipande PP, Morandi A, Hughes CG, et al. Feasibility and safety of early combined cognitive and physical therapy for critically ill medical and surgical patients: the Activity and Cognitive Therapy in ICU (ACT-ICU) trial. Intensive Care Med 2014;40:370-379.

9. Parker A, Sricharoenchai T, Needham DM. Early Rehabilitation in the Intensive Care Unit: Preventing Physical and Mental Health Impairments. Curr Phys Med Rehabil Rep 2013;1:307-314.

10. Tan T, Brett SJ, Stokes T; Guideline Development Group. Rehabilita- 
tion after critical illness: summary of NICE guidance. BMJ 2009;338: b822.

11. Ely EW, Margolin R, Francis J, May L, Truman B, Dittus R, et al. Evaluation of delirium in critically ill patients: validation of the Confusion Assessment Method for the Intensive Care Unit (CAM-ICU). Crit Care Med 2001;29:1370-1379.

12. Choi SH, Na DL, Oh KM, Park BJ. A short form of the Samsung Dementia Questionnaire (S-SDQ): development and cross-validation. J Korean Neurol Assoc 1999;17:253-258.

13. Borson S, Scanlan JM, Chen P, Ganguli M. The Mini-Cog as a screen for dementia: validation in a population-based sample. J Am Geriatr Soc 2003;51:1451-1454.

14. Park JH, Lee DW. Validity of dementia screening test 'Korean version of the Mini-Cog. J Korean Geriatr Psychiatry 2012;16:111-116.

15. Kroenke K, Spitzer RL, Williams JB. The Patient Health Questionnaire-2: validity of a two-item depression screener. Med Care 2003;41: 1284-1292.

16. Twigg E, Humphris G, Jones C, Bramwell R, Griffiths RD. Use of a screening questionnaire for post-traumatic stress disorder (PTSD) on a sample of UK ICU patients. Acta Anaesthesiol Scand 2008;52:202208.

17. Jones C, Griffiths RD, Slater T, Benjamin KS, Wilson S. Significant cognitive dysfunction in non-delirious patients identified during and persisting following critical illness. Intensive Care Med 2006;32:923926.

18. Torgersen J, Hole JF, Kvale R, Wentzel-Larsen T, Flaatten H. Cognitive impairments after critical illness. Acta Anaesthesiol Scand 2011;55: 1044-1051.

19. Robbins TW, James M, Owen AM, Sahakian BJ, McInnes L, Rabbitt P. Cambridge Neuropsychological Test Automated Battery (CANTAB): a factor analytic study of a large sample of normal elderly volunteers. Dementia 1994;5:266-281.

20. Samuelson KA, Lundberg D, Fridlund B. Stressful memories and psychological distress in adult mechanically ventilated intensive care patients - a 2-month follow-up study. Acta Anaesthesiol Scand 2007;51: 671-678. 